\title{
Phytoprotection
}

\section{Gradation et régulation de Lymantria dispar L. (Lepidoptera, Lymantriidae) avec Bacillus thuringiensis Berliner var. kurstaki dans les subéraies du centre et de l'est algérien Gradation and regulation of Lymantria dispar $L$. (Lepidoptera, Lymantriidae) with Bacillus thuringiensis Berliner var. kurstaki in centre and east Algerian cork oak forests}

\section{Mohamed Zamoum, Mohamed Khemici et Rachid Bahmane}

Volume 94, numéro 1, 2014

Reçu le 2013-12-01; accepté le 2014-03-31

URI : https://id.erudit.org/iderudit/1027062ar

DOI : https://doi.org/10.7202/1027062ar

\section{Aller au sommaire du numéro}

Éditeur(s)

Société de protection des plantes du Québec (SPPQ)

ISSN

1710-1603 (numérique)

Découvrir la revue

Citer cet article

Zamoum, M., Khemici, M. \& Bahmane, R. (2014). Gradation et régulation de Lymantria dispar L. (Lepidoptera, Lymantriidae) avec Bacillus thuringiensis Berliner var. kurstaki dans les subéraies du centre et de l'est algérien. Phytoprotection, 94(1), 13-18. https://doi.org/10.7202/1027062ar

\section{Résumé de l'article}

Lymantria dispar (Lepidoptera, Lymantriidae) est l'un des insectes les plus redoutables pour les subéraies algériennes. Le recensement des superficies infestées entre 1968 et 2013 a mis en évidence trois principaux pics de culmination en 1977, 1987 et 1996 qui ont conduit à la défoliation de 13 445, 28 157 et 20793 ha de forêts, respectivement. Les forêts littorales de l'est algérien semblent les plus vulnérables. Pour la première fois en Algérie, un essai à l'aide de deux produits à base de Bacillus thuringiensis Berliner var. kurstaki (Btk) de sérotype 3a-3b à la concentration de 16000 unités internationales par milligramme (UI/mg) a été réalisé sur les premiers stades larvaires de l'insecte dans trois foyers infestés afin de déterminer les conditions optimales d'utilisation de bio-insecticides en subéraie. Les relevés effectués 20 j après la pulvérisation par hélicoptère ont montré que la mortalité du premier au troisième stade larvaire a atteint au moins $90 \%$ dans les zones traitées. L'élevage des chenilles et des chrysalides survivantes au traitement a permis de constater une mortalité différée entre $16,0 \%$ et $38,8 \%$ dans les foyers traités. La mortalité nymphale était principalement causée par les hyménoptères, les diptères et les microorganismes pathogènes.
Tous droits réservés @ La société de protection des plantes du Québec, 2014
Ce document est protégé par la loi sur le droit d'auteur. L’utilisation des services d'Érudit (y compris la reproduction) est assujettie à sa politique d'utilisation que vous pouvez consulter en ligne.

https://apropos.erudit.org/fr/usagers/politique-dutilisation/ 


\title{
Gradation et régulation de Lymantria dispar L. (Lepidoptera, Lymantriidae) avec Bacillus thuringiensis Berliner var. kurstaki dans les subéraies du centre et de l'est algérien
}

\author{
Mohamed Zamoum ${ }^{1 凶}$, Mohamed Khemici ${ }^{1}$ et Rachid Bahmane ${ }^{2}$
}

Reçu le 2013-12-01; accepté le 2014-03-31

PHYTOPROTECTION 94 : 13-18

Lymantria dispar (Lepidoptera, Lymantriidae) est I'un des insectes les plus redoutables pour les subéraies algériennes. Le recensement des superficies infestées entre 1968 et 2013 a mis en évidence trois principaux pics de culmination en 1977, 1987 et 1996 qui ont conduit à la défoliation de 13 445, 28157 et 20793 ha de forêts, respectivement. Les forêts littorales de l'est algérien semblent les plus vulnérables. Pour la première fois en Algérie, un essai à l'aide de deux produits à base de Bacillus thuringiensis Berliner var. kurstaki (Btk) de sérotype 3a-3b à la concentration de 16000 unités internationales par milligramme $(\mathrm{UI} / \mathrm{mg})$ a été réalisé sur les premiers stades larvaires de l'insecte dans trois foyers infestés afin de déterminer les conditions optimales d'utilisation de bio-insecticides en subéraie. Les relevés effectués 20 j après la pulvérisation par hélicoptère ont montré que la mortalité du premier au troisième stade larvaire a atteint au moins $90 \%$ dans les zones traitées. L'élevage des chenilles et des chrysalides survivantes au traitement a permis de constater une mortalité différée entre 16,0\% et $38,8 \%$ dans les foyers traités. La mortalité nymphale était principalement causée par les hyménoptères, les diptères et les microorganismes pathogènes.

Mots-clés : Bacillus thuringiensis, défoliation, ennemis naturels, infestation, Lymantria dispar, Quercus suber

[Gradation and regulation of Lymantria dispar L. (Lepidoptera, Lymantriidae) with Bacillus thuringiensis Berliner var. kurstaki in centre and east Algerian cork oak forests]

Lymantria dispar (Lepidoptera, Lymantriidae) is one of the most destructive insect pests in Algerian cork oak forests. The identification of areas infested between 1968 and 2013 by this insect indicates three main peaks of culminations in 1977, 1987 and 1996 during which 13,445, 28,157 and 20,793 ha of forests were defoliated, respectively. Eastern coastal forests seem to be the most vulnerable. For the first time in Algeria, we conducted a test on first larval instars with two commercial products based on Bacillus thuringiensis Berliner var. kurstaki (Btk) serotype 3a-3b at a concentration of 16,000 International Units per milligram (IU/mg) in three infested areas in order to determine the optimal conditions for using bioinsecticides in cork oak forests. Surveys conducted $20 \mathrm{~d}$ after helicopter spraying showed that mortality from the first to the third instars reached at least $90 \%$ in the areas treated. Rearing caterpillars and pupae that survived the treatment revealed a delayed mortality of 16.0 to $38.8 \%$ in the treated areas. Pupal mortality was mainly due to Hymenoptera, Diptera and pathogenic microorganisms.

Key words: Bacillus thuringiensis, defoliation, infestation, Lymantria dispar, natural enemies, Quercus suber

1. Institut national de recherche forestière, Division de recherche en santé des forêts, B.P. 37, Chéraga Alger, Algérie; $\triangle$ mzamoum@yahoo.fr

2. Direction générale des forêts, Division de la protection des forêts, Ben Aknoun Alger, Algérie 


\section{INTRODUCTION}

Les subéraies représentent une richesse inestimable du point de vue écologique, économique, scientifique et culturel pour les pays du bassin méditerranéen. En Algérie, elles subissent une importante dégradation dont les causes sont multiples (Zamoum 2002). Du point de vue entomologique, au sein du complexe des lépidoptères défoliateurs des chênes, Lymantria dispar (Linnaeus, 1758) (Lepidoptera, Lymantriidae) est considéré comme étant l'un des ravageurs les plus dangereux. D'autres espèces défoliatrices provoquent aussi, quoique plus occasionnellement, des dégâts (Chambon et al. 1992; Chakali et al. 2002). Les plus communes sont Tortrix viridana (Linnaeus, 1758) (Lep., Tortricidae), Euproctis chrysorrhorea (Linnaeus, 1758), Orgyia trigotephras (Boisduval, 1828) (Lep., Lymantriidae), Ephesia nymphaea (Esper, 1787) et Ephesia nymphagoga (Esper, 1787) (Lep., Noctuidae).

Les pullulations périodiques de $L$. dispar ont fait I'objet d'un suivi en Algérie depuis 1923, date de la première description d'une infestation (Delassus 1925). À l'époque, on mentionnait déjà l'étendu et I'intensité des défoliations, l'importance des désagréments subis par les riverains (notamment face aux invasions des chenilles dans les habitations) ainsi que les enjeux reliés à l'état sanitaire des chênes, à la récolte de liège et aux dommages causés aux plantations forestières et fruitières. La polyphagie des chenilles, les possibilités de déplacement et le potentiel de multiplication de l'espèce rendent en effet les explosions démographiques de L. dispar particulièrement spectaculaires.

Depuis plus d'une décennie, un réseau d'avertissement a été installé en Algérie avec le concours des chercheurs et des gestionnaires forestiers. A ce jour, il n'existe pas d'études sur les possibilités d'utilisation des biopesticides par pulvérisation aérienne contre les chenilles de L. dispar dans les conditions écologiques des subéraies algériennes.

Pour répondre à cette préoccupation, un essai de traitement à l'aide de deux biopesticides homologués à base de Bacillus thuringiensis var. kurstaki (Btk) a été entrepris dans les conditions particulières des subéraies en montagne.

\section{MATÉRIEL ET MÉTHODES}

\section{Estimation des superficies infestées}

Dans le cadre du réseau national d'avertissement (RNA), les superficies infestées par $L$. dispar ont été estimées depuis 1968 dans toutes les subéraies afin de suivre les rythmes de gradation de cet insecte. Ce recensement des populations est effectué particulièrement dans les forêts de chêne-liège de Tizi Ouzou ( $N=6)$, Jijel $(N=11)$, Sétif $(N=3)$, Skikda $(N=1)$ et Annaba $(N=1)$. Dans chacune des forêts, des stations couvrant environ 100 hectares (ha) ont été choisies en fonction de leurs caractéristiques environnementales dont l'altitude, I'exposition et la topographie du site ainsi que la structure du peuplement.
La méthode utilisée consiste premièrement à dénombrer toutes les pontes de 30 arbres-échantillons par station d'observation afin d'estimer le nombre moyen de pontes par arbre et par station. Par la suite, elle consiste à mesurer la longueur d'au moins 60 pontes et classifier les taux de défoliation selon les seuils suivants : $0 \%$ (absence de défoliation), entre 1 et $10 \%$ (défoliation faible), entre 10 et $25 \%$ (défoliation légère), entre 25 et $50 \%$ (défoliation moyenne), entre 50 et $75 \%$ (défoliation forte) et entre 75 et $100 \%$ (défoliation totale).

\section{Installation des placettes expérimentales et relevés biologiques pour l'essai avec $B t k$}

Trois foyers d'infestation de $L$. dispar ont été retenus pour les essais à Mascaro (Blida), Tifrit (Tizi Ouzou) et Oued Agrioun (Béjaïa), et ce, après des observations de la présence de pontes de l'insecte en quantité suffisante pour occasionner des dégâts aux arbres infestés. Une parcelle a été tracée dans chaque foyer en tenant compte des paramètres géostationnels et de la superficie du biotope. Il s'agissait de diviser la zone en parcelles de 50 ha en fonction de la configuration du relief (haut, mi et bas versant) et de l'exposition, puis de délimiter dans chacune d'elles une placette d'observation d'environ 10 ha. Dans chacune des placettes, 30 arbres ont été choisis au hasard en suivant un ou plusieurs transects le long desquels un arbre sur cinq a été retenu au centre de la placette. Une parcelle témoin non traitée $(T)$, située loin des possibilités de dérive de l'insecticide, a également été choisie dans chacun des foyers.

Au centre de chaque forêt, 10 arbres ont été choisis en suivant un transect linéaire perpendiculaire au trajet du vol de l'hélicoptère lors des pulvérisations. Pour chaque arbre échantillonné, quatre rameaux d'environ $1 \mathrm{~m}$ de longueur ont été prélevés selon les quatre points cardinaux à la date d'intervention (I) puis à $\mathrm{I}+10 \mathrm{j}$ et à $\mathrm{I}+20 \mathrm{j}$. Au cours du premier prélèvement, la composition larvaire dans les stations a été établie (pourcentage des stades L1, L2 et L3; tableau $2)$. Pour les deux autres prélèvements $(I+10 \mathrm{j}$ et $\mathrm{I}+$ $20 \mathrm{j})$, le taux de défoliation a été estimé par le biais d'un indice de défoliation (ID). Cet indice est défini visuellement en se basant sur le pourcentage approximatif (entre 0 et $100 \%$ ) de pertes de feuilles par rapport à la biomasse foliaire totale de l'arbre échantillonné.

\section{Essai de traitement au Btk}

Deux formulations à base de Btk ont été testées dans des conditions météorologiques favorables (absence de pluie, vent de moins de $5 \mathrm{~m} / \mathrm{s}$ et température avoisinant $20^{\circ} \mathrm{C}$ ) : le Dipel $8 \mathrm{~L}$ fourni par la société Atlas Agro et le Bio T Plus fourni par Cogenal. La pulvérisation des deux biopesticides a été faite tôt le matin (à partir de 6 h00 T.U.) entre le 6 et le 8 mai 2006 en utilisant la technique " ultra bas volume " à l'aide d'un hélicoptère équipé de micronairs (AU 7000 à hélice) selon les modalités reportées dans le tableau 1. Ces formulations ont été utilisées, car elles comportent de nombreux avantages du fait que le Btk a un spectre d'action limité aux lépidoptères et qu'il atteint principalement les chenilles de $L$. dispar par une action larvicide rapide (Kwang-Bo et Côté 2000). 
Tableau 1. Modalités de traitement retenues dans chacune des trois stations traitées.

\begin{tabular}{cccccc}
\hline Localisation & $\begin{array}{c}\text { Surface } \\
\text { traitée (ha) }\end{array}$ & $\begin{array}{c}\text { Produit } \\
\text { utilisé }\end{array}$ & $\begin{array}{c}\text { Concentration } \\
\text { (UI/mg) }\end{array}$ & $\begin{array}{c}\text { Dose } \\
\text { (I/ha) }\end{array}$ & $\begin{array}{c}\text { Dates de } \\
\text { traitement }\end{array}$ \\
\hline Mascaro & 100 & Bio T Plus & 16000 & 2,5 & $08-05$ \\
Tifrit & 450 & Dipel 8L & 16000 & 3 & $07-05$ \\
Oued Agrioun & 300 & Dipel 8L & 16000 & 3 & $06-05$ \\
\hline
\end{tabular}

\section{Élevage des chenilles et des chrysalides}

Des échantillons de chrysalides et de chenilles du dernier stade larvaire de L. dispar qui ont survécu au traitement avec Btk ont été prélevés au hasard dans les parcelles traitées, puis mis en élevage au laboratoire le 16 mai 2006 à Oued Agrioun $(N=220)$, le 17 mai à Tifrit $(N=275)$ et le 18 mai à Mascaro $(N=217)$. La population témoin $(N=300)$ a été prélevée le 18 mai dans la subéraie de Chréa qui est le prolongement de la forêt de Mascaro se trouvant en versant nord du Parc national de Chréa (Blida).

Les chenilles du dernier stade ont été nourries à I'aide de feuilles de chêne-liège prélevées à proximité du laboratoire. Toutes les chrysalides ont été placées sur un lit de sciure dans des boîtes de plastique transparent mesurant $30 \mathrm{~cm} \times 20 \mathrm{~cm} \times 10 \mathrm{~cm}$. L'émergence des adultes de $L$. dispar et de ses parasitoïdes a été notée chaque jour.

\section{Analyses statistiques}

Dans les trois forêts traitées, l'effet du Btk a été estimé par la régression des effectifs moyens de chenilles à la date d'intervention (I), puis à $10 \mathrm{j}(\mathrm{I}+10)$ et $20 \mathrm{j}$ $(I+20)$ après le traitement pour chacune des placettes. Les taux de mortalité parcellaire ont été calculés à $I+10 j$ et $I+20 j$ par rapport à l'effectif moyen de chenilles avant le traitement. Les taux moyens de défoliation dans chacune des parcelles ont été calculés sur la base des données obtenues à partir des arbres échantillonnés. Les moyennes des pontes recensées ainsi que de leurs longueurs ont été comparées avec le test $t$ de Student entre chacune des forêts. Le test de $\chi^{2}$ à deux entrées multiples a été utilisé pour comparer les taux de survie et de mortalité obtenus à partir des populations ayant survécues au Btk dans les forêts traitées et dans celles non traitées.

\section{RÉSULTATS}

\section{Fluctuations des superficies infestées}

Comme cette étude le montre, les résultats du RNA ont donné un aperçu annuel de l'évolution des gradations de L. dispar et ont permis la détection des foyers d'infestation d'autres défoliateurs potentiels. En 2006, les principaux foyers de pullulation de $L$. dispar étaient signalés à Tizi Ouzou, Blida et Béjaïa. On a également recensé des infestations de Catocala nymphagoga à Tissemssilt et Aïn Defla sur Quercus ilex (Linnaeus 1753), ainsi que des infestations d'E. chrysorrhoea dans I'Akfadou sur le chêne afarès (Quercus afares) et le chêne zeen ( $Q$. canariensis).

Les surfaces défoliées enregistrées au cours des quatre dernières décennies (1968-2013) montrent que les gradations de $L$. dispar en Algérie se succèdent approximativement tous les 10 ans. L'étendue de la défoliation dans une région et pour une année donnée peut aller de quelques hectares à plus de 20000 ha (maximum observé : 23530 ha à Jijel en 1987) (Figure 1). Les défoliations dues aux autres espèces de défoliateurs sont de moindre importance et se manifestent de façon sporadique comme l'ont déjà constaté Ckakali et al. (2002).

II semble que les périodes de culmination dans les cas de Tizi Ouzou et Jijel présentent des périodes de gradation différentes. En effet, la périodicité des culminations observées est de 4 à 8 ans à Tizi Ouzou et de 9 à 10 ans à Jijel. II semble également qu'en général, durant les années 1986, 1987, 1988, 1992, 1996, 2001 et 2006, I'augmentation des superficies infestées s'est manifestée à l'échelle des régions est et centre de l'Algérie.

Par ailleurs, les résultats des recensements de pontes réalisés dans toutes les subéraies ont permis d'identifier trois foyers d'infestation de L. dispar à Mascaro (Blida), Tifrit (Tizi Ouzou) et Oued Agrioun (Béjaïa). Dans le tableau 3, les relevés de chacune des stations montrent, selon le test $t$ de Student, une différence significative pour les effectifs moyens de pontes sur les 30 arbres qui ont dépassé $50 \mathrm{~mm}$ à Mascaro et ceux compris entre 14,7 et $47 \mathrm{~mm}$ à Tifrit, puis ceux entre 8,9 et $33,2 \mathrm{~mm}$ à Oued Agrioun. II existe également une différence significative pour les longueurs moyennes des pontes entre les trois foyers à l'exception de Tifrit et Oued Agrioun.

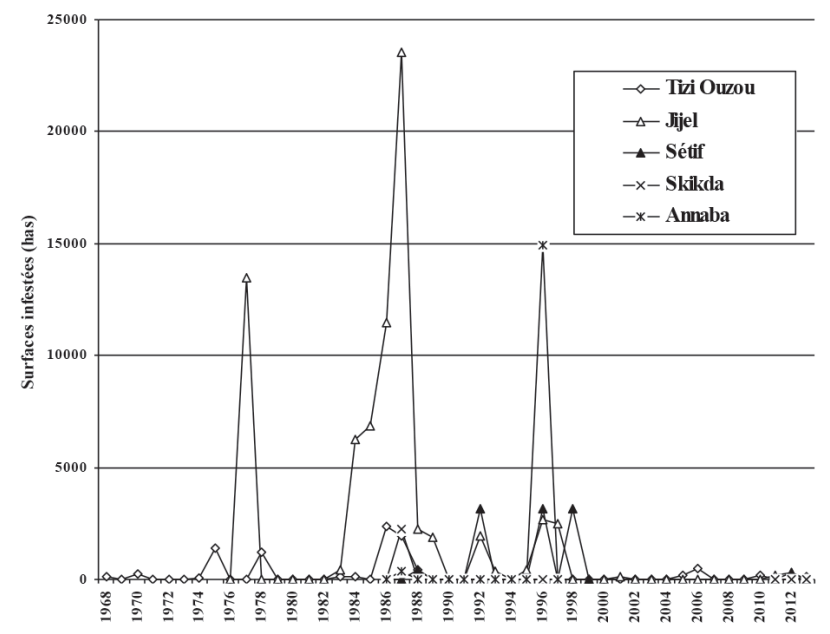

Figure 1. Variations annuelles des superficies défoliées par $L$. dispar entre 1968 et 2013 dans les principales subéraies du centre et de l'est algérien. 
Tableau 2. Abondance relative (\%) des stades larvaires L1, L2, L3 et L4 de $L$. dispar juste avant le traitement dans les trois stations expérimentales.

\begin{tabular}{ccccc}
\hline Stations & L1 & L2 & L3 & L4 \\
\hline Mascaro & 19,5 & 46,7 & 28,6 & 5,2 \\
Tifrit & 14,4 & 55,2 & 22,1 & 8,3 \\
Oued Agrioun & 17,6 & 68,8 & 10,5 & 3,1 \\
\hline
\end{tabular}

Tableau 3. Nombre moyen de pontes de $L$. dispar (N) et leurs longueurs moyennes en mm dans les trois foyers d'infestation.

\begin{tabular}{|c|c|c|c|c|c|c|c|c|c|c|c|c|c|c|c|}
\hline \multirow{2}{*}{$\frac{\text { Localités }}{\text { Altitude (m) }}$} & \multicolumn{2}{|c|}{ Mascaro } & \multicolumn{7}{|c|}{ Tifrit } & \multicolumn{6}{|c|}{ Oued Agrioun } \\
\hline & \multicolumn{2}{|c|}{$100-150$} & \multicolumn{7}{|c|}{$150-372$} & \multicolumn{6}{|c|}{$600-800$} \\
\hline Placettes & 1 & 2 & 1 & 2 & 3 & 4 & 5 & 6 & 7 & 1 & 2 & 3 & 4 & 5 & 6 \\
\hline $\mathrm{N}^{*}$ & 57,2 & 36,1 & 32,3 & 19,2 & 30,5 & 47,0 & 32,4 & 41,2 & 14,7 & 12,6 & 11,2 & 23,1 & 9,0 & 8,9 & 33,2 \\
\hline Longueurs** & 54,4 & 42,8 & 21,5 & 21,0 & 23,0 & 23,0 & 17,0 & 17,5 & 14,5 & 22,6 & 25,2 & 19,7 & 20,3 & 18,2 & 20,8 \\
\hline
\end{tabular}

* Différence significative entre les foyers d'infestation (Test $t$ de Student, $P<0,05$ ).

* Différence significative entre les foyers d'infestation (Test $t$ de Student, $P<0,05$ ), sauf entre Tifrit et Oued Agrioun $(P>0,05)$.

Tableau 4. Taux moyen de défoliation (ID) et estimation des taux de mortalité parcellaire comparés obtenus avec le Btk à partir des effectifs moyens de chenilles de $L$. dispar (N) dénombrés dans les placettes traitées et témoins (T) dans chacune des localités.

\begin{tabular}{|c|c|c|c|c|c|c|c|}
\hline \multirow[b]{2}{*}{ Localités } & \multirow[b]{2}{*}{ Placettes } & \multirow{2}{*}{\multicolumn{2}{|c|}{$\begin{array}{l}N \\
I+10\end{array}$}} & \multirow[b]{2}{*}{$1+20$} & \multicolumn{2}{|c|}{ Mortalité (\%) } & \multirow{2}{*}{$\begin{array}{l}\text { ID } \\
I+20\end{array}$} \\
\hline & & & & & $I+10$ & $I+20$ & \\
\hline \multirow{3}{*}{$\begin{array}{l}\text { Mascaro } \\
\text { (Blida) }\end{array}$} & 1 & 125,1 & 22,4 & 9,3 & 82,1 & 92,6 & 15 \\
\hline & 2 & 75,1 & 11,2 & 7,1 & 85,1 & 90,5 & 15 \\
\hline & $\mathrm{T}$ & 52,2 & 33,3 & 29,1 & 36,2 & 44,3 & 100 \\
\hline \multirow{8}{*}{$\begin{array}{c}\text { Tifrit } \\
\text { (Tizi Ouzou) }\end{array}$} & 1 & 81,4 & 9,7 & 6,3 & 88,1 & 92,3 & 10 \\
\hline & 2 & 31,9 & 24,8 & 1,7 & 22,3 & 94,7 & 10 \\
\hline & 3 & 63,2 & 24,7 & 0 & 60,9 & 100 & 5 \\
\hline & 4 & 108,2 & 21,3 & 0 & 80,3 & 100 & 5 \\
\hline & 5 & 60,0 & 24,1 & 0 & 59,8 & 100 & 5 \\
\hline & 6 & 59,6 & 20,2 & 0 & 66,1 & 100 & 15 \\
\hline & 7 & 36,4 & 12,1 & 0 & 66,8 & 100 & 10 \\
\hline & $\mathrm{T}$ & 47,3 & 28,2 & 19,1 & 40,4 & 59,6 & 100 \\
\hline \multirow{7}{*}{$\begin{array}{l}\text { Oued Agrioun } \\
\text { (Béjaïa) }\end{array}$} & 1 & 16,0 & 3,8 & 1,8 & 76,3 & 88,8 & 20 \\
\hline & 2 & 8,1 & 1,8 & 0,8 & 77,8 & 90,1 & 10 \\
\hline & 3 & 34,2 & 6,3 & 4,7 & 81,6 & 86,3 & 15 \\
\hline & 4 & 6,0 & 1 & 0 & 83,4 & 100 & 5 \\
\hline & 5 & 5,3 & 0,7 & 0 & 86,0 & 100 & 5 \\
\hline & 6 & 27,2 & 5,8 & 1 & 78,3 & 96,9 & 25 \\
\hline & $\mathrm{T}$ & 22,6 & 12,0 & 8,9 & 46,9 & 60,6 & 75 \\
\hline
\end{tabular}

Tableau 5. Incidence relative des ennemis naturels et devenir des individus ayant survécu dans les trois stations traitées (Mascaro ( $N=217$ ), Tifrit ( $N=275$ ) et Béjaïa $(N=220)$ ) et dans la station non traitée (Chréa $(N=300)$ ).

\begin{tabular}{crcr} 
Mascaro & Tifrit & Béjaïa & Chréa \\
\hline 73,9 & 61,2 & 84,0 & 31,0 \\
10,1 & 8,9 & 12,0 & 36,9 \\
0,0 & 0,0 & 0,0 & 3,6 \\
1,4 & 0,0 & 0,0 & 2,4 \\
1,4 & 0,0 & 0,0 & 1,2 \\
7,2 & 1,2 & 0,0 & 13,1 \\
2,9 & 4,2 & 0,0 & 9,5 \\
2,9 & 9,8 & 4,0 & 0,0 \\
0,0 & 14,7 & 0,0 & 2,4 \\
\hline
\end{tabular}

\footnotetext{
* Parasitoïdes non identifiés
} 


\section{Incidence des insecticides à base de Btk}

Le jour du traitement, les chenilles des stades L1 et L2 dominaient dans les populations des sites sélectionnés. Elles représentaient dans l'ensemble plus de $65 \%$ des chenilles récoltées et atteignaient $86,4 \%$ à Oued Agrioun (Tableau 2).

Les biopesticides à base de Btk ont provoqué dans l'ensemble plus de $90 \%$ de mortalité des chenilles dans toutes les placettes, à l'exception des placettes 1 et 3 de la forêt d'Ouled Agrioun (Tableau 4). Les dénombrements effectués après le traitement ont montré que les chenilles non touchées par le traitement étaient de stade L3 et du début de stade L4 et que les défoliations n'ont pas dépassé les $25 \%$.

\section{Facteurs de mortalité des chenilles et des chrysalides}

Dans le tableau 5, la proportion d'adultes obtenus à partir des chenilles âgées et de chrysalides provenant des sites traités (entre 61,2 et $84,0 \%$ ) apparaît comme étant très significativement supérieure à celle des individus récoltés la même semaine dans la forêt non traitée de Chréa (31\%) $\left(\chi^{2}=182,0 ; \mathrm{dl}=24 ; p<0,05\right)$. Les taux cumulés de parasitisme ont atteint $66,7 \%$ à Chréa alors qu'ils étaient compris entre 12,0 et $23,2 \%$ dans les placettes traitées.

Parmi les parasitoïdes obtenus dans nos élevages ${ }^{1}$, nous avons observé deux espèces de diptères, dont la tachinaire Exorista segregata (Rondani, 1859) (Dipt., Tachinidae) qui pond sur les chenilles et qui émerge au stade prénymphal ou nymphal. De plus, parmi les hyménoptères parasitoïdes de chrysalides obtenus, nous avons identifié les Ichneunonidae Pimpla turionellae (Linnaeus, 1758) et Pimpla rufipes (Miller, 1759) (= instigator F. 1793) ainsi que le Chalcididae Brachymeria tibialis intermedia (Nees, 1834).

II apparaît que l'action du virus de la polyédrose nucléaire (VPN) chez les chrysalides issues de chenilles traitées se situe entre 2,9 et 9,8 \% dans le cas des maladies (chrysalides avec altération des cellules et une odeur nauséabonde) alors qu'elle est de $14,7 \%$ pour l'entomomycose à Beauveria bassiana ([Bals-Criv.] Vuillemin, 1912). Chez les chenilles non traitées, les chrysalides présentant des symptômes de " maladie " sont absentes. Par contre, les mycoses sont présentes avec un faible taux de 2,4\%.

\section{DISCUSSION}

La récapitulation des données relatives aux superficies infestées par L. dispar montre qu'à l'exception des subéraies de Tizi Ouzou et Jijel, il est nécessaire de considérer qu'il n'est pas certain que toutes les défoliations d'une région donnée aient été recensées, surtout si elles étaient de très petite taille. Ces lacunes ont des causes diverses, mais la plus importante est liée à la difficulté de détecter les foyers primaires lorsqu'ils ne dépassent pas quelques hectares ou qu'ils sont situés dans des secteurs peu accessibles, contrainte que l'on retrouve dans la majorité des subéraies. A Tizi Ouzou, il est probable que les

1. Identifiés par Mme Villemant, Musée national d'histoire naturelle de Paris (France). petits pics de culmination observés à quelques années de différence soient des parties d'une même gradation générale. Cet écart est probablement dû à une différence entre les subéraies de plaines et les subéraies d'altitude. En plaine, la défoliation s'étend sur plusieurs années et sur de très grandes surfaces, tandis qu'en montagne, les forêts d'altitude sont défoliées les unes après les autres sur une période assez longue.

Les culminations observées à tous les 10 ans à Jijel, avec des invasions sur de vastes superficies, ont également été notées dans le cas de la subéraie marocaine par Villemant et Fraval (1999). Ces explosions démographiques peuvent être expliquées par divers facteurs déclencheurs, dont ceux en relation avec I'aptitude des ennemis naturels à réguler les effectifs de L. dispar (Hamra Kroua 1986; Khous 1993) et ceux en rapport avec le climat (Martinat 1987). Par contre, en Kabylie, la fréquence élevée des attaques de cet insecte ravageur pourrait être facilitée par les déséquilibres de la structure des subéraies de cette région qui sont touchées par les incendies et plusieurs délits anthropiques (surpâturage, ébranchage, coupe de bois et exploitation anarchique du liège).

Les résultats obtenus confirment la performance du Btk, un biopesticide d'ingestion très sélectif ayant une bonne incidence sur le contrôle des effectifs de populations de L. dispar. Son efficacité a été démontrée dans d'autres conditions écologiques à travers I'aire de répartition de L. dispar au Maroc (Fraval et al. 1977), au sud de l'Europe (Luciano et al. 2003; Martin et al. 2002) et en Amérique du Nord (Andreadis et al. 1982; Jobin et Caron 1982; Yendol et al. 1973).

Par ailleurs, l'étude comparée des populations de $L$. dispar ayant survécu au traitement et des populations non traitées a permis d'évaluer l'incidence des différents facteurs de régulation pouvant intervenir après la pulvérisation de Btk. En effet, il semble que la mortalité due aux ennemis naturels se maintienne dans une certaine mesure chez les populations traitées, plus particulièrement celle causée par les mycoses et par le VPN déjà identifié sur ce ravageur par Khous et al. (1991). Ce virus a déjà été observé chez d'autres espèces de lépidoptères comme Thaumetopoea pityocampa (Denis et Schiffermüller 1775; Zamoum et al. 2005) et E. chrysorrhoea (Ruelle et al. 1977).

Les taux de parasitisme obtenus pour $P$. instigator à Chréa $(3,6 \%)$ sont plus élevés que ceux observés à Tikjda (entre 0,2 et 0,3\%) par Khous (1993), probablement en raison de la collecte d'une population de L. dispar au stade de chrysalide à Chréa sur lequel $P$. instigator est actif. L'absence du Pimplinae dans les sites traités est peut-être due aux problèmes liés à la coïncidence temporelle entre le parasitoïde et son hôte. En effet, le ralentissement du développement larvaire chez les chenilles traitées au Btk a été démontré en laboratoire (Cerboneschi 2002). De plus, la présence élevée de chrysalides " malades " chez les populations traitées limite l'activité parasitaire de I'ichneumonidae (Carton 1975, cité par Khous 1993).

En ce qui concerne $B$. intermedia, I'un des parasitoïdes nymphaux les plus efficaces, il semble que les taux de parasitisme obtenus dans les foyers traités sont non négligeables (entre 8,9 et $12 \%$ ). Dans la subéraie de Annaba, l'incidence de cette espèce n'a 
été que de 1,05\% en phase de latence (Hamra Kroua 1986); à Tikjda, elle a été entre 6,9 et 34,2 \% (Khous 1993) en phase de culmination. À Chréa, le taux a dépassé $36,9 \%$. Il a été démontré que l'activité de ce parasitoïde est plus importante lors des défoliations et de l'ouverture du peuplement (Barbosa et al. 1978). En protégeant le feuillage par le biais des arrosages au Btk, il est probable que l'aptitude parasitaire de cette espèce soit limitée après le traitement.

Comme dans le cas des autres parasitoïdes polyphages, la tachinaire $E$. segregata semble avoir une action variable qui est très vraisemblablement en relation avec la richesse de la biodiversité qui permet la présence d'hôtes de transition. L'incidence de $8,4 \%$ d'E. segregata dans les foyers traités semble être plus importante à Mascaro par rapport aux autres forêts. Néanmoins, sa faible incidence par rapport à Chréa $(13,1 \%)$ pourrait être induite par la présence de chenilles infectées par le Btk à la suite du traitement et des microorganismes pathogènes (Luciano et Prota 1986). II apparaît aussi que l'incidence des ennemis naturels semble contribuer à une mortalité différée chez les populations ayant survécu au traitement; elle peut aller de 16 à 38,8 \% .

À l'issue de ce bio-essai, il a été possible de démontrer qu'il existe une alternative pour contrôler les chenilles de $L$. dispar dans les conditions écologiques des subéraies algériennes. Par contre, les interventions au Btk ne peuvent être intéressantes que dans les situations où l'on observe des niveaux élevés de population de $L$. dispar, lesquels peuvent provoquer une défoliation sévère et engendrer un affaiblissement des peuplements et ainsi occasionner leur dépérissement. Cette défoliation peut (1) être accompagnée d'un incident climatique majeur, comme une période de sécheresse prolongée; (2) survenir à l'occasion de programmes d'exploitation de liège; (3) avoir cours dans de jeunes plantations de chênes en période de vulnérabilité; ou (4) survenir dans des conditions urbaines ou récréatives où l'aspect esthétique peut être affecté. Par ailleurs, les interventions au Btk peuvent être préconisées, de façon préventive, dans des foyers primaires d'infestation afin d'éviter ou de ralentir la propagation de $L$. dispar et pour favoriser un renforcement du complexe des ennemis naturels lors d'un désynchronisme avec leur hôte.

\section{REMERCIEMENTS}

Les auteurs remercient C. Villemant (Musée national $\mathrm{d}^{\prime}$ histoire naturelle de Paris, France) pour son aide à I'amélioration du manuscrit et à l'identification des parasitoïdes, J.C. Martin (INRA, Avignon, France) pour ses suggestions et la Direction générale des forêts d'Algérie pour les moyens mis à notre disposition pour réaliser cette étude.

\section{RÉFÉRENCES}

Andreadis, T.G., N.R. Dubois, R.M. Weseloh, R.E.B. Moore, J.F. Anderson et F.B. Lewis. 1982. Aerial spray tests with Bacillus thuringiensis for control of the gypsy moth in Connecticut. Conn. Agric. Exp. Stn. Bull. No. 807, 5 pp.
Barbosa, P., E.A. Frongillo et W. Cranshaw. 1978. Orientation of field populations of Brachymeria intermedia [Hym.: Chalcidae] to host and habitat cues. Entomophaga 23 : 63-67.

Cerboneschi, A. 2002. Effets sublétaux de Bacillus thuringiensis var. kurstaki sur le développement du lépidoptère Lymantria dispar (L.) infecté à différents stades larvaires. IOBC/WPRS Bull. 25 : 123-130.

Chakali, G., A. Attal-Bedreddine et H. Ouzani 2002. Les insectes ravageurs des chênes, Quercus suber et $Q$. ilex, en Algérie. IOBC/WPRS Bull. 25: 93-100.

Chambon, J.P., M.G. Khous, G. Genestier et C. Pineau. 1992. Contribution à l'inventaire des lépidoptères des forêts (chênaies et cédraies) d'Algérie. Ann. Rech. For. Algérie $2: 44-84$.

Delassus, M. 1925. La lutte contre le Liparis dispar en Algérie. Rev. Agric. Afr. Nord 23 : 334-352.

Fraval, A., F. Herard, M. Jarry, P. Questienne, M.C. Viland et D. Vein. 1977. Note sur un traitement au moyen d'une préparation de Bacillus thuringiensis Berliner contre Porthetria dispar L. en forêt de la Mamora (Maroc). Ann. Rech. For. Maroc 17 : 67-76.

Hamra Kroua, S. 1986. Note préliminaire sur les ennemis naturels de Lymantria dispar (L.) dans les forêts de chêne-liège du Nord constantinois. Ann. Inst. Nat. Agro. (El-Harrach) $10: 26-44$.

Jobin, L.J. et A. Caron. 1982. Résultats de traitements aériens à I'aide du Dimilin et du Bacillus thuringiensis pour combattre la spongieuse Lymantria dispar L. au Québec. Rev. rech. Serv. can. for. $3: 11-13$.

Khous, M.G., J.R. Valéro et L. Jobin. 1991. La présence d'une polyédrose nucléaire chez Lymantria dispar (L.) en Algérie. Biocontrol News 4 : 54-57.

Khous, M.G. 1993. Contribution à l'étude de l'écobiologie et du contrôle naturel du Lymantria dispar (L.) (Lepidoptera : Lymantriidae) en chenaie verte du Djurdjura (Tikjda). Thèse de maîtrise en entomologie forestière, Université des sciences et de la technologie Houari Boumediene, Alger, Algérie, $133 \mathrm{pp}$.

Kwang-Bo, J. et J.C. Côté 2000. Une analyse des incidences environnementales de l'insecticide microbien Bacillus thuringiensis. Bull. tech. $\mathrm{n}^{\circ} 29$, Centre de R\&D en horticulture, Saint-Jean-sur-Richelieu, Qc, $17 \mathrm{pp}$.

Luciano, P. et R. Prota. 1986. La dinamica di popolazione di Lymantria dispar L. in Sardegna. III. Indicatori biologici della gradazione. Frustula Entomol. 7-8 : 613-630.

Luciano, P., A. Lentini et O.V. Cao. 2003. La lotta ai lepidotteri defogliatori delle sugherete in provincia di Sassari. Industria grafica Poddighe, Sassari, $69 \mathrm{pp}$.

Martin, J.C., C. Villemant et R. Mazet. 2002. Utilisation de Bacillus thuringiensis var. kurstaki 3a-3b dans la lutte contre le bombyx disparate et problèmes posés par les migrations de chenilles. IOBC/WPRS Bull. 25 : 115-122.

Martinat, P.J. 1987. The role of climatic variation and weather in forest insect outbreaks. Pages 241-268 in P. Barbosa et J.C. Schultz (éds.), Insect Outbreaks. Academic Press, Londres, G.-B.

Villemant, C. et A. Fraval. 1999. Les gradations de Lymantria dispar en Europe et en Afrique du Nord. IOBC/WPRS Bull. $22: 71-79$

Ruelle, P., L. Nef et P. Lebrun. 1977. Étude en laboratoire de l'efficacité prolongée de Bacillus thuringiensis sur Euproctis chrysorrhoea. Parasitica 33 : 127-137.

Yendol, W.C., Hamlen R.A., F.B. Lewis. 1973. Evaluation of Bacillus thuringiensis for gypsy moth suppression. J. Econ. Entomol. 66 : 183-186.

Zamoum, M. 2002. Quelques éléments pour la préservation de la santé des forêts en Algérie. Rev. For. Alg. 4 : 4-7.

Zamoum, M., G. Démolin, J.C. Martin et A. Bensidi. 2005. Lutte contre la processionnaire du pin. Que deviennent les chenilles survivantes après un traitement $B t$ aux stades L3 et L4. Phytoma 585 : 38-41. 